\title{
ANY ADMISSIBLE HARMONIC RITZ VALUE SET IS POSSIBLE FOR GMRES*
}

\author{
KUI DU ${ }^{\dagger}$, JURJEN DUINTJER TEBBENS $\ddagger$, AND GÉRARD MEURANT ${ }^{\S}$
}

\begin{abstract}
It is known that the harmonic Ritz values are the zeros of the GMRES residual polynomials. In this paper we show that any decreasing GMRES residual norm history is possible with any prescribed finite harmonic Ritz values in every iteration of the GMRES process. In addition, we characterize the admissible harmonic Ritz values when GMRES stagnates during some iterations, and show that with any prescribed, in this sense admissible harmonic Ritz values, any non-increasing GMRES residual norm history is possible. We also present a parameterization of the class of matrices and right-hand sides yielding prescribed GMRES residual norms and prescribed admissible harmonic Ritz values.
\end{abstract}

Key words. Ritz values, harmonic Ritz values, GMRES convergence, prescribed residual norms, FOM convergence

AMS subject classifications. 65F15, 65F10

1. Introduction. The Generalized Minimum Residual (GMRES) iterative method due to Saad and Schultz [41] is one of the most popular Krylov methods for solving large, sparse, nonsingular and non-Hermitian linear systems $A x=b$. During several decades, intensive research has been invested in convergence analysis of GMRES. The convergence behavior of Hermitian counterparts of GMRES like the CG [23] and MINRES methods [37] can well be explained by the distribution of the eigenvalues of $A$. An important question in GMRES convergence analysis is to determine to what extent eigenvalues influence the convergence of the method.

Mathematically, the GMRES convergence may be governed not only by the eigenvalues of $A$. This was shown very clearly in a series of papers by Arioli, Greenbaum, Pták and Strakoš $[1,21,22]$. These papers show that for any chosen sequence of positive, non-increasing numbers, there exists a class of right-hand sides and matrices, whose nonzero eigenvalues can be prescribed, giving residual norms that coincide with the given non-increasing sequence. GMRES residual norm convergence curves are, in this sense, independent of the eigenvalues of $A$. In [31] closed-form expressions for the GMRES residual norms were derived as functions of the eigenvalues and eigenvectors of $A$ as well as the right-hand side $b$. These expressions show precisely how the residual norms depend on the eigenvalues, the pairwise differences of eigenvalues and on the eigenvectors (or principal vectors if $A$ is not diagonalizable). In a nutshell, when the matrix $A$ is normal, the dependence of the residual norms on the eigenvectors is mild. But in the general case, GMRES convergence may depend strongly on the eigenvectors in addition to the eigenvalues of $A$. Several papers look for approaches other than eigenvalue analysis to explain GMRES convergence. Some examples are approaches based on the field of values [12], potential theory [26], pseudospectra [35, 46], the polynomial numerical hull [20], comparison with GMRES for non-Euclidean inner products [39] or decomposition into normal plus low-rank [24]. Even if they can be very suited for particular problems, none of the approaches appears to represent a universal tool to explain the convergence behavior of the GMRES method.

${ }^{*}$ Received February 2, 2017. Accepted May 11, 2017. Published online on September 18, 2017. Recommended by K. Jbilou.

${ }^{\dagger}$ School of Mathematical Sciences and Fujian Provincial Key Laboratory of Mathematical Modeling and High Performance Scientific Computing, Xiamen University, Xiamen 361005, China (kuidu@ xmu . edu . cn).

${ }^{\ddagger}$ Institute of Computer Science, Academy of Sciences of the Czech Republic, Pod Vodárenskou věží 2, 18207 Praha 8 - Libeň and Charles University, Faculty of Pharmacy in Hradec Králové, Heyrovského 1203, 50005 Hradec Králové, Czech Republic (duint jertebbens@cs.cas.cz).

$\S 30$ rue du sergent Bauchat, 75012 Paris, France (gerard.meurant@gmail.com). 
The GMRES method uses the Arnoldi process [2] to compute an orthonormal basis of the Krylov subspace. The Arnoldi process generates upper Hessenberg matrices $H_{k}$ whose eigenvalues (the so-called Ritz values) are often used as approximations of the eigenvalues of $A$. In the CG method there is a strong correlation between the convergence of Ritz values to eigenvalues and the convergence of CG-iterates to the solution of the linear system [47]. Another relevant question for GMRES convergence is to know how the (potential) convergence of the Ritz values is related to the GMRES residual norms; see, i.e., [48]. An answer was given in [9] where it was shown that one can construct right-hand sides and matrices with prescribed eigenvalues, prescribed GMRES residual norms and prescribed Ritz values at every GMRES iteration.

The convergence speed of the GMRES method in practice is often such that the method needs to be accelerated somehow. While the most popular way to do so is through the use of a preconditioner, significant attention has been paid to so-called deflation techniques (which may be incorporated into preconditioning themselves). Based on spectral information about $A$ which is obtained during the GMRES process, the influence of eigenvalues which are assumed to hamper convergence is eliminated. Among a large number of proposed deflation techniques, some examples are described in $[3,6,7,8,14,15,17,18,25,27,28,32,33,34,38]$. Even if, in general, no eigenvalues might hamper convergence at all according to the results by Arioli, Greenbaum, Pták and Strakoš [1, 21, 22], deflation methods have shown to be able to accelerate GMRES in many applications. Information about the spectrum of $A$ is extracted, during the GMRES process, from either the Ritz values or from the harmonic Ritz values (though some deflation methods are more focused on Ritz vectors in relation with multigrid techniques $[43,44])$. Harmonic Ritz values are mostly preferred over ordinary Ritz values and this may have two main reasons: First, harmonic Ritz values are related to an Arnoldi process involving $A^{-1}$ and appear to be more suited to find eigenvalues close to zero than ordinary Ritz values. Precisely eigenvalues close to zero are often assumed to be responsible for slow GMRES convergence. Second, harmonic Ritz values are more strongly connected to GMRES residual norms: Every GMRES residual vector is the product of a polynomial in $A$ with the initial residual vector and the roots of this residual polynomial are the harmonic Ritz values [36]. The ordinary Ritz values are in fact the roots of the residual polynomials of the Full Orthogonalization method (FOM) [40], which is closely related to GMRES but which does not minimize the residual norms [19]. One therefore expects that the extent to which GMRES residual norms are independent of ordinary Ritz values is not the same for harmonic Ritz values; the latter should be strongly influencing residual norms because of their immediate relation with the GMRES residual polynomial. In this paper we show that, surprisingly, GMRES residual norms are independent of harmonic Ritz values as well. More precisely, our main result shows that one can construct right-hand sides and matrices with prescribed eigenvalues, prescribed GMRES residual norms and prescribed harmonic Ritz values at every GMRES iteration.

This paper is organized as follows. In the next section, we introduce some notation and define harmonic Ritz values sets that are admissible with respect to GMRES residual norms. Section 3 addresses FOM residual norms in relation with ordinary Ritz values. Section 4 contains the main result of the paper on prescribed GMRES residual norms in combination with prescribed harmonic Ritz values. In Section 5 we illustrate the result with some small numerical examples and Section 6 contains closing remarks. To facilitate the discussion, we shall adopt the following notation. For a matrix $A$, let $a_{i j}, A_{k}, \underline{A}_{k}$, and $A^{*}$ denote the $i, j$ entry, the upper-left $k \times k$ submatrix, the upper-left $(k+1) \times k$ submatrix, and the conjugate transpose of $A$, respectively. The complex conjugate of a scalar $z$ is $\bar{z}$, and $e_{k}$ represents the $k$ th column of the identity matrix of appropriate order. 
2. Preliminaries. Let a nonsingular matrix $A \in \mathbb{C}^{n \times n}$ and a vector $b \in \mathbb{C}^{n}$ be given. For an initial guess $x_{0} \in \mathbb{C}^{n}$, GMRES [41] approximates the exact solution of $A x=b$ at step $k$ by the vector $x_{k} \in x_{0}+\mathcal{K}_{k}\left(A, r_{0}\right)$ that minimizes the Euclidean norm of the residual $r_{k}=b-A x_{k}$, i.e.,

$$
\left\|r_{k}\right\|=\min _{x \in x_{0}+\mathcal{K}_{k}\left(A, r_{0}\right)}\|b-A x\|=\min _{v \in \mathcal{K}_{k}\left(A, r_{0}\right)}\left\|r_{0}-A v\right\|,
$$

where the Krylov subspace is defined as

$$
\mathcal{K}_{k}\left(A, r_{0}\right)=\operatorname{span}\left\{r_{0}, A r_{0}, \ldots, A^{k-1} r_{0}\right\} .
$$

Due to this minimization property, the residual vector $r_{k}$ is the orthogonal projection of $r_{0}$ onto $\left(A \mathcal{K}_{k}\left(A, r_{0}\right)\right)^{\perp}$, the orthogonal complement of $A \mathcal{K}_{k}\left(A, r_{0}\right)$. It can therefore be uniquely expressed as

$$
r_{k}=p_{k}(A) r_{0},
$$

where $p_{k}(z)$ is the GMRES residual polynomial of the $k$ th iteration. It is of degree at most $k$ and has the value one at the origin; see, for example, [45].

The Arnoldi process [2] constructs an orthonormal basis of $\mathcal{K}_{k}\left(A, r_{0}\right)$. We assume that the Arnoldi process for the pair $\left\{A, r_{0}\right\}$ does not break down before the $n$th iteration. Then after $n$ iterations the process has generated the Arnoldi decomposition

$$
A V=V H,
$$

where $V$ is unitary and $H$ is irreducible upper Hessenberg. The $k$ eigenvalues $\left\{\mu_{j}^{(k)}\right\}_{j=1}^{k}$ of the eigenvalue problem

$$
H_{k} w=\mu w
$$

are called Ritz values at step $k$ of the Arnoldi process for $\left\{A, r_{0}\right\}$. We will rather call them ordinary Ritz values to clearly distinguish them from harmonic Ritz values. The $k$ eigenvalues $\left\{\theta_{j}^{(k)}\right\}_{j=1}^{k}$ of the generalized eigenvalue problem

$$
\underline{H}_{k}^{*} \underline{H}_{k} w=\theta H_{k}^{*} w, \quad k<n,
$$

are called harmonic Ritz values at step $k$ of the Arnoldi process for $\left\{A, r_{0}\right\}$. We do not consider here harmonic Ritz values resulting from a shift of the original spectrum to find close to zero eigenvalues, i.e., we always assume implicitly zero shifts. Because $H$ is irreducible upper Hessenberg, $\underline{H}_{k}^{*} \underline{H}_{k}$ is non-singular and thus no harmonic Ritz value $\theta_{j}^{(k)}$ can be zero. However, when $H_{k}$ is singular, some of the harmonic Ritz values $\theta_{j}^{(k)}$ are infinite.

LEMMA $2.1([5,16])$. The GMRES residual polynomial $p_{k}(z)$ in $(2.1)$ can be expressed as

$$
p_{k}(z)=\prod_{j=1}^{k}\left(1-\frac{z}{\theta_{j}^{(k)}}\right),
$$

where $\left\{\theta_{j}^{(k)}\right\}_{j=1}^{k}$ are the harmonic Ritz values at step $k$.

THEOREM 2.2. Let $\Theta^{(k)}$ denote the $k$-tuple of the (not necessarily mutually distinct) harmonic Ritz values at step $k$ :

$$
\Theta^{(k)}=\left(\theta_{1}^{(k)}, \theta_{2}^{(k)}, \ldots, \theta_{k}^{(k)}\right) .
$$


If GMRES applied to $\left\{A, r_{0}\right\}$ stagnates from step $k+1$ to step $k+m(k+m \leq n-1)$, i.e.,

$$
\left\|r_{k}\right\|=\left\|r_{k+1}\right\|=\cdots=\left\|r_{k+m}\right\|
$$

then, for $i=1: m$, the $(k+i)$-tuple of the harmonic Ritz values at step $k+i$ is

$$
\Theta^{(k+i)}=\left(\theta_{1}^{(k)}, \theta_{2}^{(k)}, \ldots, \theta_{k}^{(k)}, \infty, \cdots, \infty\right) .
$$

Proof. Because $r_{k}$ is the orthogonal projection of $r_{0}$ onto $\left(A \mathcal{K}_{k}\left(A, r_{0}\right)\right)^{\perp}, r_{k+1}$ is the orthogonal projection of $r_{0}$ onto $\left(A \mathcal{K}_{k+1}\left(A, r_{0}\right)\right)^{\perp}$, and because $A \mathcal{K}_{k}\left(A, r_{0}\right) \subseteq A \mathcal{K}_{k+1}\left(A, r_{0}\right)$, $\left\|r_{k}\right\|=\left\|r_{k+1}\right\|$ implies $r_{k}=r_{k+1}$. Therefore, $\left\|r_{k}\right\|=\left\|r_{k+1}\right\|=\cdots=\left\|r_{k+m}\right\|$ implies $r_{k}=r_{k+1}=\cdots=r_{k+m}$.

It follows from $r_{k}=r_{k+1}=\cdots=r_{k+m}$ and the uniqueness of the GMRES residual polynomial that

$$
p_{k}(z)=p_{k+1}(z)=\cdots=p_{k+m}(z) .
$$

Then the statement of the theorem is a direct consequence of Lemma 2.1.

Theorem 2.2 shows that if one prescribes GMRES to have some stagnating iterations, this puts conditions on the corresponding harmonic Ritz values. We will show later that these are the only conditions which prescribed residual norms can impose. Even if the proof follows in a later section, we give the definition of admissible harmonic Ritz values with respect to chosen residual norms already discussed here.

DEFINITION 2.3. For prescribed GMRES residual norms

$$
\left\|r_{0}\right\| \geq\left\|r_{1}\right\| \geq \cdots \geq\left\|r_{n-1}\right\| \geq\left\|r_{n}\right\|=0,
$$

we call a set of tuples of nonzero complex and infinite numbers

$$
\begin{aligned}
\Theta & =\left\{\Theta^{(1)}, \Theta^{(2)}, \ldots, \Theta^{(n)}\right\} \\
& =\left\{\theta_{1}^{(1)},\left(\theta_{1}^{(2)}, \theta_{2}^{(2)}\right), \cdots,\left(\theta_{1}^{(n)}, \theta_{2}^{(n)}, \ldots, \theta_{n}^{(n)}\right)\right\}
\end{aligned}
$$

an admissible harmonic Ritz value set if the tuples in $\Theta$ satisfy the property in Theorem 2.2.

We have here, in contrast with (2.2), considered the harmonic Ritz values $\Theta^{(n)}$ for the $n$th iteration. The convention is that they coincide with the ordinary Ritz values for the $n$th iteration and thus with the eigenvalues of $A$. Before showing that admissible harmonic Ritz values can be chosen independently of GMRES residual norms we take a look at the somewhat simpler but analogue problem for ordinary Ritz values and FOM residual norms.

3. Ordinary Ritz values and the FOM method. For an initial guess $x_{0}$, FOM approximates the exact solution of $A x=b$ at step $k$ by the vector $x_{k}^{F} \in x_{0}+\mathcal{K}_{k}\left(A, r_{0}\right)$, such that the residual $r_{k}^{F}=b-A x_{k}^{F}$ is orthogonal to $\mathcal{K}_{k}\left(A, r_{0}\right)$, i.e.,

$$
r_{k}^{F} \perp \mathcal{K}_{k}\left(A, r_{0}\right) .
$$

This translates to FOM iterates being of the form

$$
x_{k}^{F}=x_{0}+V^{(k)} y^{(k)},
$$

where $y^{(k)}$ is obtained by solving

$$
H_{k} y^{(k)}=\left\|r_{0}\right\| e_{1},
$$


and $V^{(k)}$ consists of the first $k$ columns of $V$. Then, the residual is $r_{k}^{F}=-h_{k+1, k} e_{k}^{T} y^{(k)} v_{k+1}$ and the norm of the residual vector is

$$
\left\|r_{k}^{F}\right\|=h_{k+1, k}\left|e_{k}^{T} y^{(k)}\right| .
$$

The latter relation holds in fact for any Q-OR method [13].

As mentioned in the introduction, the roots of the FOM residual polynomial $p_{k}^{F}$ such that $r_{k}^{F}=p_{k}^{F}(A) r_{0}$ (with $p_{k}^{F}(0)=1$ ) are the ordinary Ritz values at iteration $k$ [19]. Nevertheless, as we will show next, the FOM residual norm can be fully independent of the corresponding Ritz values. To gain more insight into the FOM residual norm, let us analyze the entries of $y^{(k)}$ in (3.2). The matrix $H$ in (2.2) can be factored as

$$
H=U^{-1} C U,
$$

where $U$ is upper triangular with real positive entries on the diagonal and $C$ is the companion matrix of the eigenvalues of $H$ (and of $A$ ); see for instance [29] or [10] where the factorization process is described. Note that

$$
h_{j+1, j}=\frac{u_{j, j}}{u_{j+1, j+1}}, j=1, \ldots, n-1,
$$

with $u_{i, j}$ denoting the entries of $U$ with $u_{1,1}=1 /\left\|r_{0}\right\|$. The matrix $U$ is linked to the Krylov matrix

$$
K=\left[r_{0}, A r_{0}, \ldots, A^{n-1} r_{0}\right]
$$

and to the matrix of the basis vectors $V$ by $K U=V$; see, e.g., [9, 10, 11].

The principal submatrix $H_{k}$ of $H$ in (3.2) can be factored in a similar way with a companion matrix revealing the eigenvalues of $H_{k}$, which are the ordinary Ritz values. This was done in [11]. However, since the notation we use here is a little bit different, we reformulate this result.

THEOREM 3.1. The upper Hessenberg matrix $H_{k}$ computed in the Arnoldi process, for $k<n$, can be factored as

$$
H_{k}=U_{k}^{-1} C^{(k)} U_{k},
$$

where $U_{k}$ is the principal submatrix of order $k$ of $U$ obtained from the Krylov matrix $K$ and the unitary matrix $V$ through $K U=V$, and $C^{(k)}$ is the companion matrix for the Ritz values at iteration $k$. Moreover,

$$
C^{(k)}=E_{k}+\left[\begin{array}{llll}
0 & \cdots & 0 & -\frac{1}{u_{k+1, k+1}} U_{1: k, k+1}
\end{array}\right],
$$

where $E_{k}$ is a square down-shift matrix of order $k$,

$$
E_{k}=\left[\begin{array}{ccccc}
0 & & & & \\
1 & 0 & & & \\
& \ddots & \ddots & & \\
& & 1 & 0 & \\
& & & 1 & 0
\end{array}\right]
$$

and $U_{1: k, k+1}$ is the vector of the first $k$ components of the $(k+1)$ st column of $U$. 
Proof. Clearly

$$
H_{k}=\left[\begin{array}{ll}
I_{k} & 0
\end{array}\right] H\left[\begin{array}{c}
I_{k} \\
0
\end{array}\right]=\left[\begin{array}{ll}
I_{k} & 0
\end{array}\right] U^{-1} C U\left[\begin{array}{c}
I_{k} \\
0
\end{array}\right],
$$

where $I_{k}$ is the identity matrix of order $k$. Let

$$
\left[\begin{array}{ll}
I_{k} & 0
\end{array}\right] U^{-1}=\left[\begin{array}{ll}
U_{k}^{-1} & Z
\end{array}\right], \quad U\left[\begin{array}{c}
I_{k} \\
0
\end{array}\right]=\left[\begin{array}{c}
U_{k} \\
0
\end{array}\right] .
$$

Since $C=E_{n}+\left[\begin{array}{llll}0 & \cdots & 0 & s\end{array}\right]$, it yields

$$
H_{k}=\left[\begin{array}{ll}
U_{k}^{-1} & Z
\end{array}\right]\left[\begin{array}{c}
E_{k} U_{k} \\
e_{1} e_{k}^{T} U_{k}
\end{array}\right]=U_{k}^{-1} E_{k} U_{k}+Z e_{1} e_{k}^{T} U_{k} .
$$

The vector $Z e_{1}$ of length $k$ is made of the first $k$ components of the $(k+1)$ st column of $U^{-1}$, and is $-U_{k}^{-1} U_{1: k, k+1} / u_{k+1, k+1}$. Factoring $U_{k}^{-1}$ on the left and $U_{k}$ on the right gives the result.

Theorem 3.1 shows that the coefficients of the characteristic polynomial $\pi_{k}(\lambda)=\lambda^{k}+$ $\alpha_{k-1} \lambda^{k-1}+\cdots+\alpha_{0}$ of $H_{k}$ are

$$
\left[\begin{array}{c}
\alpha_{0} \\
\vdots \\
\alpha_{k-1}
\end{array}\right]=\frac{1}{u_{k+1, k+1}} U_{1: k, k+1}=\frac{1}{u_{k+1, k+1}}\left[\begin{array}{c}
u_{1, k+1} \\
\vdots \\
u_{k, k+1}
\end{array}\right] .
$$

The roots of the polynomial $\pi_{k}$ are the ordinary Ritz values at iteration $k$. From Theorem 3.1 we also have for $y^{(k)}$ in (3.1), assuming that $H_{k}$ is nonsingular,

$$
y^{(k)}=\left\|r_{0}\right\| H_{k}^{-1} e_{1}=\left\|r_{0}\right\| U_{k}^{-1}\left[C^{(k)}\right]^{-1} U_{k} e_{1}=U_{k}^{-1}\left[C^{(k)}\right]^{-1} e_{1} .
$$

If we write the companion matrix $C^{(k)}$ as

$$
C^{(k)}=\left[\begin{array}{cc}
0 & -\alpha_{0} \\
I_{k-1} & -\hat{\alpha}
\end{array}\right]
$$

with $\alpha_{0} \neq 0$ since we assumed $H_{k}$ to be nonsingular, then the inverse of the companion matrix is

$$
\left[C^{(k)}\right]^{-1}=\left[\begin{array}{cc}
-\hat{\alpha} / \alpha_{0} & I_{k-1} \\
-1 / \alpha_{0} & 0
\end{array}\right]
$$

Therefore

$$
e_{k}^{T} y^{(k)}=-\frac{1}{\alpha_{0} u_{k, k}}=-\frac{u_{k+1, k+1}}{u_{k, k} u_{1, k+1}}=-\frac{1}{u_{1, k+1} h_{k+1, k}} .
$$

Using (3.3), we have for iteration $k$, where FOM iterates exist,

$$
\left\|r_{k}^{F}\right\|=\frac{1}{\left|u_{1, k+1}\right|} .
$$

This result was proved with a more complicated proof in [11] and holds for any Q-OR method. If $H_{k}$ is singular, then $\alpha_{0}=0$ and so is $u_{1, k+1}$. 
We remark that $u_{k+1, k+1}, \ldots, u_{1, k+1}$ are the coefficients (in descending order of powers) of another polynomial whose roots are the ordinary Ritz values at the $k$ th iteration of the Arnoldi process. This holds as well for the FOM (or Q-OR) residual polynomial $p_{k}^{F}$ which is scaled such that $p_{k}^{F}(0)=1$. As a by-product of Theorem 3.1 we have that the coefficients of $p_{k}^{F}$ are

$$
\left[\begin{array}{c}
1 \\
\frac{u_{2, k+1}}{u_{1, k+1}} \\
\vdots \\
\frac{u_{k, k+1}}{u_{1, k+1}} \\
\frac{u_{k+1, k+1}}{u_{1, k+1}}
\end{array}\right]=\frac{1}{u_{1, k+1}}\left[\begin{array}{c}
u_{1, k+1} \\
\vdots \\
u_{k+1, k+1}
\end{array}\right] .
$$

If we write $u_{1, k+1}$ as $u_{1, k+1}=\left|u_{1, k+1}\right| e^{\phi_{k+1} i}$, then

$$
\left[\begin{array}{c}
u_{1, k+1} \\
\vdots \\
u_{k+1, k+1}
\end{array}\right]=\frac{1}{\left\|r_{k}^{F}\right\|} e^{\phi_{k+1} i}\left[\begin{array}{c}
1 \\
\frac{u_{2, k+1}}{u_{1, k+1}} \\
\vdots \\
\frac{u_{k, k+1}}{u_{1, k+1}} \\
\frac{u_{k+1, k+1}}{u_{1, k+1}}
\end{array}\right] .
$$

Thus the matrix $U_{k+1}$ can be factorized as

$$
U_{k+1}=\hat{U}_{k+1} D_{k+1}, \quad D_{k+1}=\left[\begin{array}{llll}
\frac{1}{\left\|r_{0}\right\|} & & & \\
& \frac{e^{\phi_{2}}}{\left\|r_{1}^{F}\right\|} & & \\
& & \ddots & \\
& & & \frac{e^{\phi_{k+1}}}{\left\|r_{k}^{F}\right\|}
\end{array}\right],
$$

and the non-zero entries of the columns of $\hat{U}_{k+1}$ are the coefficients of the residual polynomials whose roots are the ordinary Ritz values. Hence, the entries of $\hat{U}_{k+1}$ depend only on the ordinary Ritz values for the iterations 1 to $k$. If the matrix, the right-hand side and the starting vector are real, the coefficients $e^{\phi_{j} i}$ are just \pm 1 .

The above shows that it is straightforward to construct linear systems with prescribed FOM residual norms and, at the same time, prescribed ordinary Ritz values. An example is the linear system $H x=e_{1}$ where $H$ is computed according to (3.4) with $U$ being of the form

$$
U=\hat{U}_{n} D_{n},
$$

where the $k$ th column of $\hat{U}_{n}$ is chosen to be the unique FOM polynomial whose roots are the ordinary Ritz values prescribed for the $k$ th iteration. $D_{n}$ is chosen to contain the prescribed FOM residual norms, with arbitrary angles $\phi_{j}$. Other examples result from choosing a unitary matrix $Q$ and considering the system $Q^{*} H Q x=Q^{*} e_{1}$.

4. Prescribed GMRES residual norms and harmonic Ritz values. We have seen in the previous section that arbitrary ordinary Ritz values can be generated during the Arnoldi process. We now show a relation between ordinary and harmonic Ritz values in terms of the decomposition (3.5) that was used to generate arbitrary ordinary Ritz values. If GMRES does not stagnate at step $k$, i.e., $\left\|r_{k}\right\|<\left\|r_{k-1}\right\|$, then the harmonic Ritz values defined in (2.2) can also be seen as the eigenvalues of the matrix

$$
\hat{H}_{k}=H_{k}+h_{k+1, k}^{2} H_{k}^{-*} e_{k} e_{k}^{T},
$$


since $H_{k}$ is nonsingular. This follows from multiplication of (2.2) with $H_{k}^{-*}$ from the left. The following theorem was proved in [30]. For the convenience of the reader we give the proof again using the notation of this paper.

THEOREM 4.1. For $k<n$ assume that $H_{k}$ is nonsingular. Then the matrix $\hat{H}_{k}$ defined in (4.1) can be written as $\hat{H}_{k}=U_{k}^{-1} \hat{C}^{(k)} U_{k}$, with $U_{k}$ being upper triangular,

$$
\hat{C}^{(k)}=C^{(k)}-\frac{1}{u_{k+1, k+1} \overline{u_{1, k+1}}} U_{k} U_{k}^{*} e_{1} e_{k}^{T}
$$

being a companion matrix where $C^{(k)}$ is the companion matrix in $H_{k}=U_{k}^{-1} C^{(k)} U_{k}$.

Proof. Let us first consider $H_{k}^{-*} e_{k}$. We have $H_{k}^{-*}=U_{k}^{*}\left[C^{(k)}\right]^{-*} U_{k}^{-*}$. Since $U_{k}$ is upper triangular we obtain $U_{k}^{-*} e_{k}=\frac{1}{u_{k, k}} e_{k}$ with $u_{k, k}$ real and positive. Using (3.7) and taking the (Hermitian) transpose, we have

$$
\left[C^{(k)}\right]^{-*} e_{k}=-\frac{1}{\bar{\alpha}_{0}} e_{1}
$$

Finally we obtain

$$
H_{k}^{-*} e_{k}=-\frac{1}{u_{k, k} \bar{\alpha}_{0}} U_{k}^{*} e_{1} .
$$

On the other hand, we have $h_{k+1, k}=u_{k, k} / u_{k+1, k+1}$. Then

$$
\hat{H}_{k}=U_{k}^{-1} C^{(k)} U_{k}-\frac{u_{k, k}}{u_{k+1, k+1}^{2} \bar{\alpha}_{0}} U_{k}^{*} e_{1} e_{k}^{T} .
$$

Let us factor $U_{k}^{-1}$ on the left and $U_{k}$ on the right. We obtain

$$
\hat{H}_{k}=U_{k}^{-1}\left[C^{(k)}-\frac{u_{k, k}}{u_{k+1, k+1}^{2} \bar{\alpha}_{0}} U_{k} U_{k}^{*} e_{1} e_{k}^{T} U_{k}^{-1}\right] U_{k} .
$$

We remark that $e_{k}^{T} U_{k}^{-1}=\frac{1}{u_{k, k}} e_{k}^{T}$. Hence $\hat{H}_{k}$ is similar to the matrix

$$
\hat{C}^{(k)}=C^{(k)}-\frac{1}{u_{k+1, k+1}^{2} \bar{\alpha}_{0}} U_{k} U_{k}^{*} e_{1} e_{k}^{T}=C^{(k)}-\frac{1}{u_{k+1, k+1} \overline{u_{1, k+1}}} U_{k} U_{k}^{*} e_{1} e_{k}^{T},
$$

where we used (3.6). The second term on the right-hand side modifies only the last column. Therefore, $\hat{C}^{(k)}$ is a companion matrix.

COROLLARY 4.2. Under assumptions of Theorem 4.1, the coefficients of the characteristic polynomial of $\hat{H}_{k}$, whose roots are the harmonic Ritz values, are given by the negative of the last column of $\hat{C}^{(k)}$, that is, by

$$
-\hat{C}^{(k)} e_{k}=\frac{1}{u_{k+1, k+1}}\left[\begin{array}{c}
u_{1, k+1} \\
\vdots \\
u_{k, k+1}
\end{array}\right]+\frac{1}{u_{k+1, k+1} \overline{u_{1, k+1}}} U_{k} U_{k}^{*} e_{1} .
$$

Before we formulate the main theorem, we need the following auxiliary results.

LEMMA 4.3. For $k<n$, the GMRES residual norms $\left\|r_{k}\right\|$ and $\left\|r_{k-1}\right\|$ satisfy

$$
\left|u_{1, k+1}\right|^{2}=\frac{1}{\left\|r_{k}\right\|^{2}}-\frac{1}{\left\|r_{k-1}\right\|^{2}}, \quad\left\|r_{k}\right\|=\left(\sum_{l=1}^{k+1}\left|u_{1 l}\right|^{2}\right)^{-1 / 2}
$$


Proof. Using the well-known relationship

$$
\frac{1}{\left\|r_{k}^{F}\right\|^{2}}=\frac{1}{\left\|r_{k}\right\|^{2}}-\frac{1}{\left\|r_{k-1}\right\|^{2}}
$$

between the FOM and GMRES residual norms [4] and using (3.8), we immediately obtain the first claim. Then

$$
\sum_{l=1}^{k+1}\left|u_{1 l}\right|^{2}=\frac{1}{\left\|r_{k}\right\|^{2}}-\frac{1}{\left\|r_{k-1}\right\|^{2}}+\frac{1}{\left\|r_{k-1}\right\|^{2}}-\frac{1}{\left\|r_{k-2}\right\|^{2}}+\cdots+\frac{1}{\left\|r_{0}\right\|^{2}}=\frac{1}{\left\|r_{k}\right\|^{2}}
$$

THEOREM 4.4. Let

$$
\Theta=\left\{\theta_{1}^{(1)},\left(\theta_{1}^{(2)}, \theta_{2}^{(2)}\right), \cdots,\left(\theta_{1}^{(n)}, \theta_{2}^{(n)}, \ldots, \theta_{n}^{(n)}\right)\right\}
$$

be any admissible harmonic Ritz value set for prescribed GMRES residual norms $\rho_{k}, 0 \leq k<$ $n$, where

$$
\rho_{0} \geq \rho_{1} \geq \cdots \geq \rho_{n-1}>0
$$

are any non-increasing sequence of real positive numbers. Let $H=U^{-1} C U$ where $C$ is the companion matrix with eigenvalues $\theta_{1}^{(n)}, \theta_{2}^{(n)}, \ldots, \theta_{n}^{(n)}$, and the upper triangular matrix $U$ is constructed as follows. Let $u_{11}=1 /\left\|r_{0}\right\|$. For $1 \leq k<n$, if $\rho_{k}<\rho_{k-1}$, then let

$$
\begin{aligned}
& u_{1, k+1}=\frac{\beta_{0}}{\left|\beta_{0}\right|} \sqrt{1 / \rho_{k}^{2}-1 / \rho_{k-1}^{2}}, \\
& u_{k+1, k+1}=\frac{1}{\left|\beta_{0}\right| \rho_{k}^{2} \sqrt{1 / \rho_{k}^{2}-1 / \rho_{k-1}^{2}}}, \\
& u_{j, k+1}=\beta_{j-1} u_{k+1, k+1}-\frac{e_{j}^{T} U_{k} U_{k}^{*} e_{1}}{\overline{u_{1, k+1}}}, \quad j=2, \ldots, k,
\end{aligned}
$$

where $\beta_{j}, 0 \leq j \leq k-1$, are the coefficients of the polynomial

$$
\lambda^{k}+\beta_{k-1} \lambda^{k-1}+\cdots+\beta_{1} \lambda+\beta_{0}
$$

with roots $\left\{\theta_{j}^{(k)}\right\}_{j=1}^{k}$. If $\rho_{k}=\rho_{k-1}$, let $u_{1, k+1}=0, u_{k+1, k+1}$ be an arbitrarily chosen positive real number, and $u_{j, k+1}, 2 \leq j \leq k$, be arbitrarily chosen complex numbers. Then GMRES applied to $\left\{H,\left\|r_{0}\right\| e_{1}\right\}$ generates the residuals $r_{k}$ with $\left\|r_{k}\right\|=\rho_{k}$ and the harmonic Ritz value set $\Theta$.

Proof. By the construction of the first row of $U$ and using Lemma 4.3, the residual $r_{k}$ of the GMRES applied to $\left\{H, e_{1}\right\}$ satisfies $\left\|r_{k}\right\|=\rho_{k}$.

If $\rho_{k}<\rho_{k-1}$, by (4.2), straightforward calculations yield

$$
-\hat{C}^{(k)} e_{k}=\left[\begin{array}{c}
\beta_{0} \\
\vdots \\
\beta_{k-1}
\end{array}\right] .
$$

Therefore, the harmonic Ritz values at step $k$ are $\left\{\theta_{j}^{(k)}\right\}_{j=1}^{k}$. 
If $\rho_{k}=\rho_{k-1}$, according to Theorem 2.2, the harmonic Ritz values at step $k$ are then $\left\{\theta_{1}^{(k-1)}, \theta_{2}^{(k-1)}, \cdots, \theta_{k-1}^{(k-1)}, \infty\right\}$. We now show why this is true with any choice of $u_{j, k+1}$, $2 \leq j \leq k$, and any positive real choice of $u_{k+1, k+1}$. By construction, we have

$$
\underline{H}_{k}=U_{k+1}^{-1}\left[\begin{array}{c}
0 \\
I_{k}
\end{array}\right] U_{k}
$$

For simplicity, we write

$$
U_{k+1}=\left[\begin{array}{cc}
U_{k} & t \\
0 & s
\end{array}\right], \quad t=\left[\begin{array}{l}
0 \\
\hat{t}
\end{array}\right]
$$

Then

$$
H_{k}=U_{k}^{-1}\left[\begin{array}{cc}
0 & 0 \\
I_{k-1} & -\hat{t} / s
\end{array}\right] U_{k}
$$

and

$$
\underline{H}_{k}^{*} \underline{H}_{k}=U_{k}^{*}\left[\begin{array}{cc}
{\left[\begin{array}{cc}
0 & I_{k-1}
\end{array}\right] U_{k}^{-*} U_{k}^{-1}\left[\begin{array}{c}
0 \\
I_{k-1}
\end{array}\right]} & -\left[\begin{array}{ll}
0 & I_{k-1}
\end{array}\right] U_{k}^{-*} U_{k}^{-1} t / s \\
-(t / s)^{*} U_{k}^{-*} U_{k}^{-1}\left[\begin{array}{c}
0 \\
I_{k-1}
\end{array}\right] & \left(t^{*} U_{k}^{-*} U_{k}^{-1} t+1\right) / s^{2}
\end{array}\right] U_{k} .
$$

Thus, the harmonic Ritz values at step $k$ are the eigenvalues of the generalized eigenvalue problem

$$
\left[\begin{array}{cc}
{\left[\begin{array}{ll}
0 & I_{k-1}
\end{array}\right] U_{k}^{-*} U_{k}^{-1}\left[\begin{array}{c}
0 \\
I_{k-1}
\end{array}\right]} & -\left[\begin{array}{cc}
0 & I_{k-1}
\end{array}\right] U_{k}^{-*} U_{k}^{-1} t / s \\
-(t / s)^{*} U_{k}^{-*} U_{k}^{-1}\left[\begin{array}{c}
0 \\
I_{k-1}
\end{array}\right] & \left(t^{*} U_{k}^{-*} U_{k}^{-1} t+1\right) / s^{2}
\end{array}\right] y=\theta\left[\begin{array}{cc}
0 & I_{k-1} \\
0 & -\hat{t}^{*} / s
\end{array}\right] U_{k}^{-*} U_{k}^{-1} y
$$

Multiplying by $\left[\begin{array}{ll}I_{k-1} & 0 \\ \hat{t}^{*} / s & 1\end{array}\right]$ on both sides yields

$$
\left[\begin{array}{cc}
{\left[\begin{array}{cc}
0 & I_{k-1}
\end{array}\right] U_{k}^{-*} U_{k}^{-1}\left[\begin{array}{c}
0 \\
I_{k-1}
\end{array}\right]} & -\left[\begin{array}{cc}
0 & I_{k-1}
\end{array}\right] U_{k}^{-*} U_{k}^{-1} t / s \\
0 & 1 / s^{2}
\end{array}\right] y=\theta\left[\begin{array}{cc}
0 & I_{k-1} \\
0 & 0
\end{array}\right] U_{k}^{-*} U_{k}^{-1} y
$$

Therefore, the set of harmonic Ritz values at step $k$ consists of one infinite value and the eigenvalues of the generalized eigenvalue problem

$$
\left[\begin{array}{ll}
0 & I_{k-1}
\end{array}\right] U_{k}^{-*} U_{k}^{-1}\left[\begin{array}{c}
0 \\
I_{k-1}
\end{array}\right] \tilde{y}=\theta\left[\begin{array}{ll}
0 & I_{k-1}
\end{array}\right] U_{k}^{-*} U_{k}^{-1}\left[\begin{array}{c}
I_{k-1} \\
0
\end{array}\right] \tilde{y}
$$

Straightforward calculations yield

$$
\begin{aligned}
{\left[\begin{array}{ll}
0 & I_{k-1}
\end{array}\right] U_{k}^{-*} U_{k}^{-1}\left[\begin{array}{c}
I_{k-1} \\
0
\end{array}\right] } & =\left[U_{k}^{-1}\left[\begin{array}{c}
0 \\
I_{k-1}
\end{array}\right]\right]^{*} U_{k}^{-1}\left[\begin{array}{c}
I_{k-1} \\
0
\end{array}\right] \\
& =\left[U_{k}^{-1}\left[\begin{array}{c}
0 \\
I_{k-1}
\end{array}\right]\right]^{*}\left[\begin{array}{c}
I_{k-1} \\
0
\end{array}\right] U_{k-1}^{-1} \\
& \left.=\left[\begin{array}{ll}
I_{k-1} & 0
\end{array}\right] U_{k}^{-1}\left[\begin{array}{c}
0 \\
I_{k-1}
\end{array}\right]\right]^{*} U_{k-1}^{-1} \\
& =\left[U_{k-1}^{-1} C^{(k-1)}\right]^{*} U_{k-1}^{-1} \\
& =\left[C^{(k-1)}\right]^{*} U_{k-1}^{-*} U_{k-1}^{-1}
\end{aligned}
$$


By (4.4), $H_{k-1}=U_{k-1}^{-1} C^{(k-1)} U_{k-1}$, and

$$
\underline{H}_{k-1}^{*} \underline{H}_{k-1}=U_{k-1}^{*}\left[\begin{array}{ll}
0 & I_{k-1}
\end{array}\right] U_{k}^{-*} U_{k}^{-1}\left[\begin{array}{c}
0 \\
I_{k-1}
\end{array}\right] U_{k-1},
$$

and we know that the eigenvalues of the generalized eigenvalue problem (4.3) are $\left\{\theta_{j}^{(k-1)}\right\}_{j=1}^{k-1}$. This completes the proof.

THEOREM 4.5. Let $\Theta,\left\{\rho_{k}\right\}_{k=0}^{n-1}$ and $H$ be given as in Theorem 4.4. Then GMRES applied to $\left\{A, r_{0}\right\}$ generates the residuals $r_{k}$ with $\left\|r_{k}\right\|=\rho_{k}$ and the harmonic Ritz value set $\Theta$ if and only if there exists a unitary matrix $Q$ such that $A=Q H Q^{*}$ and $r_{0}=\rho_{0} Q e_{1}$.

Proof. If GMRES applied to $\left\{A, r_{0}\right\}$ generates the residuals $r_{k}$ with $\left\|r_{k}\right\|=\rho_{k}$, we have the Arnoldi decomposition $A V=V H$ and $H=U^{-1} C U$, where $C$ is the companion matrix with eigenvalues $\theta_{1}^{(n)}, \theta_{2}^{(n)}, \ldots, \theta_{n}^{(n)}$, and $U$ is upper triangular with real positive diagonal entries. For $1 \leq k<n$, we know from Lemma 4.3 that

$$
\left|u_{1, k+1}\right|=\sqrt{1 / \rho_{k}^{2}-1 / \rho_{k-1}^{2}} .
$$

If $\rho_{k}<\rho_{k}$, we write

$$
u_{1, k+1}=\sqrt{1 / \rho_{k}^{2}-1 / \rho_{k-1}^{2}} e^{\phi_{k+1} i} .
$$

Let $\beta_{j}, 0 \leq j \leq k-1$, be the coefficients of the polynomial

$$
\lambda^{k}+\beta_{k-1} \lambda^{k-1}+\cdots+\beta_{1} \lambda+\beta_{0}
$$

with roots $\left\{\theta_{j}^{(k)}\right\}_{j=1}^{k}$. As the coefficients $\beta_{j}$ coincide with the entries of $-\hat{C}^{(k)} e_{k}$, equating the first row in (4.2) and using Lemma 4.3 gives

$$
\begin{aligned}
\beta_{0} & =\frac{u_{1, k+1}}{u_{k+1, k+1}}+\frac{e_{1}^{T} U_{k} U_{k}^{*} e_{1}}{u_{k+1, k+1} \overline{u_{1, k+1}}} \\
& \left.=\frac{\sqrt{1 / \rho_{k}^{2}-1 / \rho_{k-1}^{2} e^{\phi_{k+1} i}}}{u_{k+1, k+1}}+\frac{1 / \rho_{k-1}^{2}}{u_{k+1, k+1} \sqrt{1 / \rho_{k}^{2}-1 / \rho_{k-1}^{2}} \overline{e^{\phi_{k+1} i}}}\right) e^{\phi_{k+1} i} \\
& =\left(\frac{\sqrt{1 / \rho_{k}^{2}-1 / \rho_{k-1}^{2}}}{u_{k+1, k+1}}+\frac{1 / \rho_{k-1}^{2}}{u_{k+1, k+1} \sqrt{1 / \rho_{k}^{2}-1 / \rho_{k-1}^{2}}}\right. \\
& =\frac{e^{\phi_{k+1} i}}{u_{k+1, k+1} \rho_{k}^{2} \sqrt{1 / \rho_{k}^{2}-1 / \rho_{k-1}^{2}}} .
\end{aligned}
$$

Therefore, $\phi_{k+1}$ must be the angle of $\beta_{0}$, i.e.,

$$
e^{\phi_{k+1} i}=\beta_{0} /\left|\beta_{0}\right|
$$

and then the first and $(k+1)$ st entry of $U e_{k+1}$ satisfy

$$
\begin{aligned}
& u_{1, k+1}=\frac{\beta_{0}}{\left|\beta_{0}\right|} \sqrt{1 / \rho_{k}^{2}-1 / \rho_{k-1}^{2}}, \\
& u_{k+1, k+1}=\frac{1}{\left|\beta_{0}\right| \rho_{k}^{2} \sqrt{1 / \rho_{k}^{2}-1 / \rho_{k-1}^{2}}} .
\end{aligned}
$$


The entries $u_{j, k+1}$ follow from the corresponding rows in equation (4.2):

$$
u_{j, k+1}=\beta_{j-1} u_{k+1, k+1}-\frac{e_{j}^{T} U_{k} U_{k}^{*} e_{1}}{\overline{u_{1, k+1}}}, \quad j=2, \ldots, k .
$$

If $\rho_{k}=\rho_{k-1}$, then $u_{1, k+1}=0$. As proved in Theorem 4.4, $u_{k+1, k+1}$ can be an arbitrary positive real number and $u_{i, k+1}, 2 \leq j \leq k$ can be arbitrary complex numbers.

Conversely, if there exists a unitary matrix $Q$ such that $A=Q H Q^{*}$ and $r_{0}=\rho_{0} Q e_{1}$, by the invariance under unitary similarity transformations of residual norms as well as generated Hessenberg matrices (see, for example, [45]) and Theorem 4.4, GMRES applied to $\left\{A, r_{0}\right\}$ generates the residuals $r_{k}$ with $\left\|r_{k}\right\|=\rho_{k}$ and the harmonic Ritz value set $\Theta$.

The last two theorems represent a parameterization of the class of matrices and right-hand sides generating, when GMRES is applied to a member of the class, prescribed residual norms and prescribed admissible harmonic Ritz values. The freedom left over when prescribing these quantities simultaneously, is in the unitary similarity transformations of the linear system with $Q$ (which incorporates as well the choice of the phase angles of the first row of $U$ ). If stagnation is prescribed, then there is additional freedom in the choice of the non-zero entries of the corresponding row of $U$ - they can be chosen arbitrarily except for the diagonal entry which must be positive real.

5. Numerical illustration. We have proved that any GMRES convergence history is possible with any admissible harmonic Ritz values in every iteration. In order to illustrate the strength of this result we describe a few extreme situations. We consider a very small but not fully academic example and first show that in this example harmonic Ritz values explain the behavior of GMRES much better than ordinary Ritz values.

Consider the one-dimensional convection-diffusion problem on the unit interval $[0,1]$

$$
-\nu u^{\prime \prime}+u^{\prime}=f, \quad u(0)=u(1)=0,
$$

discretized with finite differences on a regular grid with $n$ inner nodes using upwind differences for the convective term. This gives a linear system where the system matrix $A$ is tridiagonal with entries

$$
A=h^{-2} \operatorname{tridiag}(-\nu-h, 2 \nu+h,-\nu), \quad h=1 /(n+1) ;
$$

see, e.g., [42, Section 4]. In the convection dominated case, $\nu \ll h^{2}$ and $A$ is close to a scaled transposed Jordan block and in particular non-normal. Let the source term $f$ be nonzero only around the first inner node $1 /(n+1)$, with the value one at that node. Then the right-hand side $b$ is $e_{1}$. If we have $\nu=0.001$ and $n=5$, the matrix $A$ is

$$
A=\left[\begin{array}{ccccc}
6.072 & -0.036 & 0 & 0 & 0 \\
-6.036 & 6.072 & -0.036 & 0 & 0 \\
0 & -6.036 & 6.072 & -0.036 & 0 \\
0 & 0 & -6.036 & 6.072 & -0.036 \\
0 & 0 & 0 & -6.036 & 6.072
\end{array}\right], \quad\left\|W^{*} W-I_{5}\right\| \approx 3.953
$$

where $W$ denotes the normalized eigenvector matrix of $A$ ( $A$ is diagonalizable but not normal). GMRES generates the residual norms

(5.1) $\left\|r_{0}\right\|=1, \quad\left\|r_{1}\right\|=0.7050, \quad\left\|r_{2}\right\|=0.5751, \quad\left\|r_{3}\right\|=0.4978, \quad\left\|r_{4}\right\|=0.4451$.

The convergence is slow and clearly sublinear. If ordinary Ritz values influence GMRES convergence speed as suggested in [48], then we expect similar slow convergence (or possibly 
divergence) of the ordinary Ritz values. The ordinary Ritz values for the individual iterations are represented with circles in Figures 5.1 and 5.2. We see that, on the contrary, they seem to converge already in the very first step, where an exact eigenvalue was found. The second iteration finds two more eigenvalues and further iterations show smooth convergence reminding of interlacing eigenvalues for Hermitian matrices.
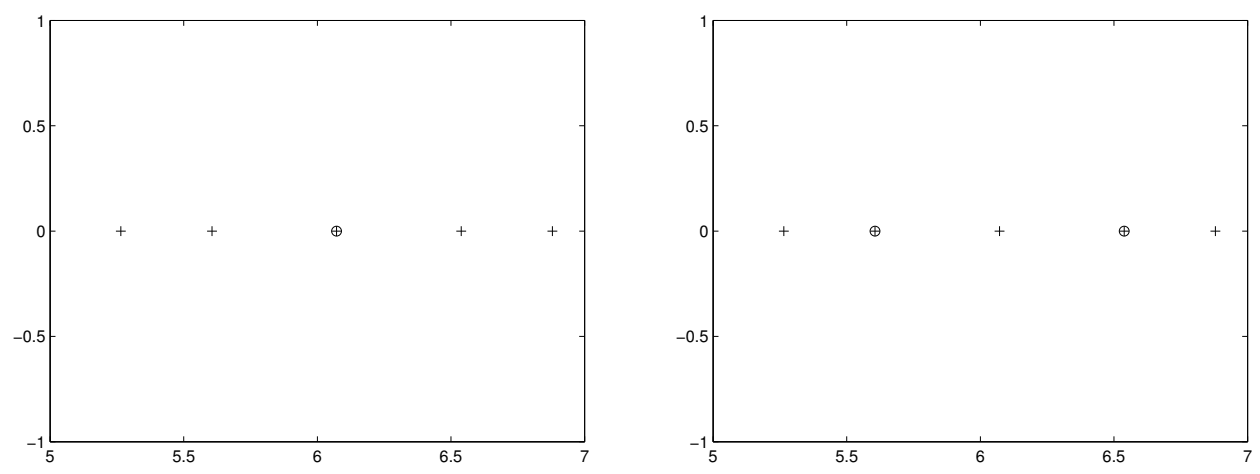

FIG. 5.1. Spectrum (plusses) and ordinary Ritz, values (circles) in iterations one (left) and two (right) with GMRES applied to $A$ and $e_{1}$.
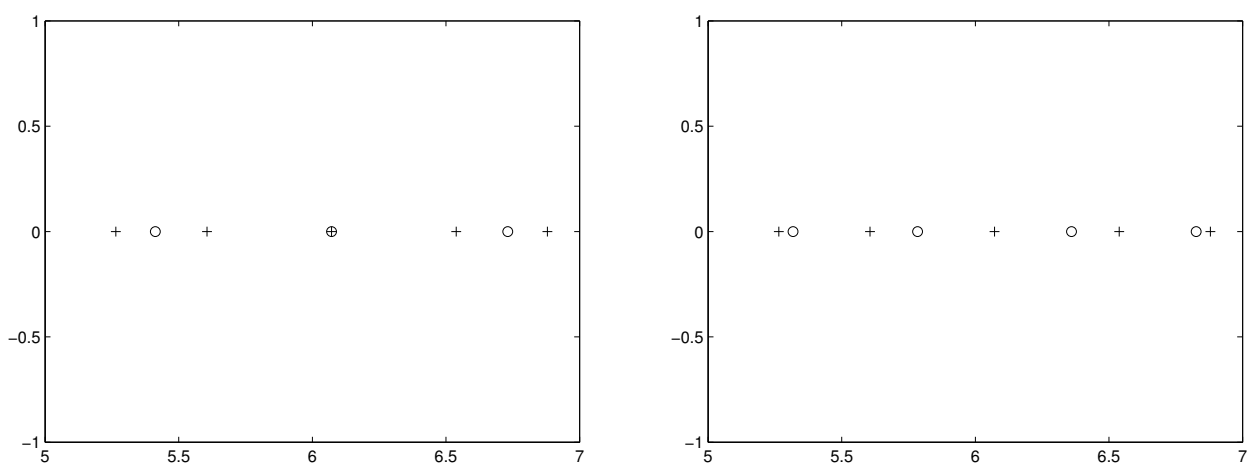

FIG. 5.2. Spectrum (plusses) and ordinary Ritz values (circles) in iterations three (left) and four (right) with GMRES applied to $A$ and $e_{1}$.

The behavior of the harmonic Ritz values, on the other hand, corresponds much better to that of GMRES in this example. The harmonic Ritz values for the individual iterations are shown in Figures 5.3 and 5.4. They stay away from the eigenvalues for all iterations and do not even seem to converge to other values, but 'dance' around the spectrum.

This example seems to indicate that the convergence of harmonic Ritz values is, in general, closely related to that of GMRES. But as we have proved in the previous sections, this is not the case. We can for instance, using Theorem 4.1, generate another upper Hessenberg matrix $H$ such that it generates with the right-hand side $e_{1}$ the same residual norms (5.1) as $A$ but with harmonic Ritz values that are identical with the nicely converging ordinary Ritz values in 

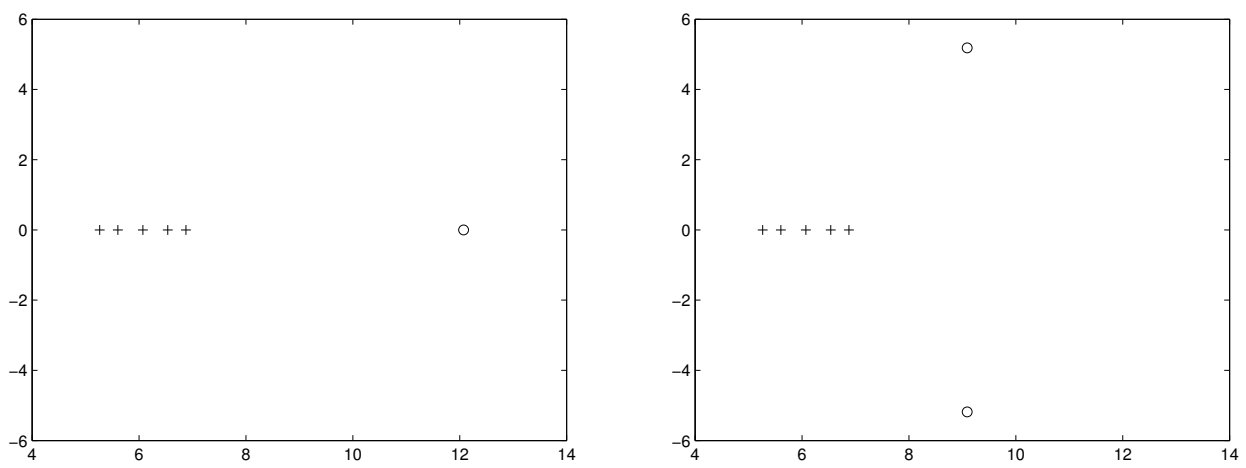

FIG. 5.3. Spectrum (plusses) and harmonic Ritz values (circles) in iterations one (left) and two (right) with GMRES applied to $A$ (or $\tilde{H})$ and $e_{1}$.
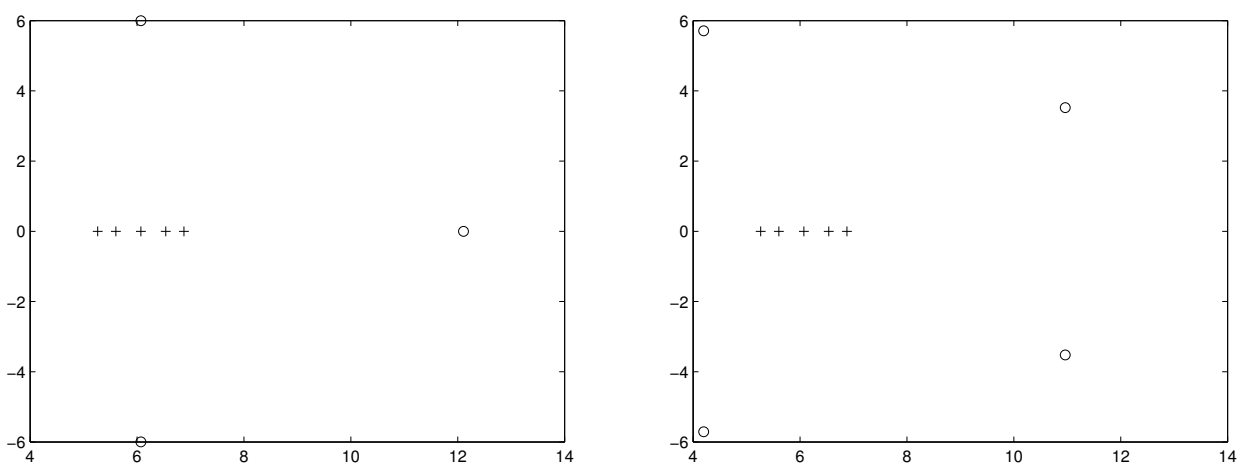

FIG. 5.4. Spectrum (plusses) and harmonic Ritz values (circles) in iterations three (left) and four (right) with GMRES applied to $A($ or $\tilde{H})$ and $e_{1}$.

Figures 5.1 and 5.2. This matrix $H$ is

$$
H=\left[\begin{array}{ccccc}
3.054 & 1.064 & -0.486 & 0.296 & 4.8 \\
3.036 & 5.073 & 0.560 & -0.297 & -4.829 \\
0 & 4.016 & 5.566 & 0.351 & 4.829 \\
0 & 0 & 4.522 & 5.769 & -4.781 \\
0 & 0 & 0 & 4.826 & 10.898
\end{array}\right], \quad\left\|W^{*} W-I_{5}\right\| \approx 3.988
$$

where we rounded to three decimal places; the matrix is not much less normal than $A$. The ordinary Ritz values of this matrix $H$ are displayed in Figures 5.5 and 5.6. They seem to be, except for an outlier, often close to the exact eigenvalues in spite of slow GMRES convergence.

There is no reason either why the harmonic Ritz values would not behave, with the same residual norms (5.1), in a counter-intuitive manner midway between perfect convergence and plain divergence. For instance, they could have converged in step one, diverge in step two, converge in step three and diverge again in the last step. An example of this behavior is given in Figures 5.7 and 5.8. 

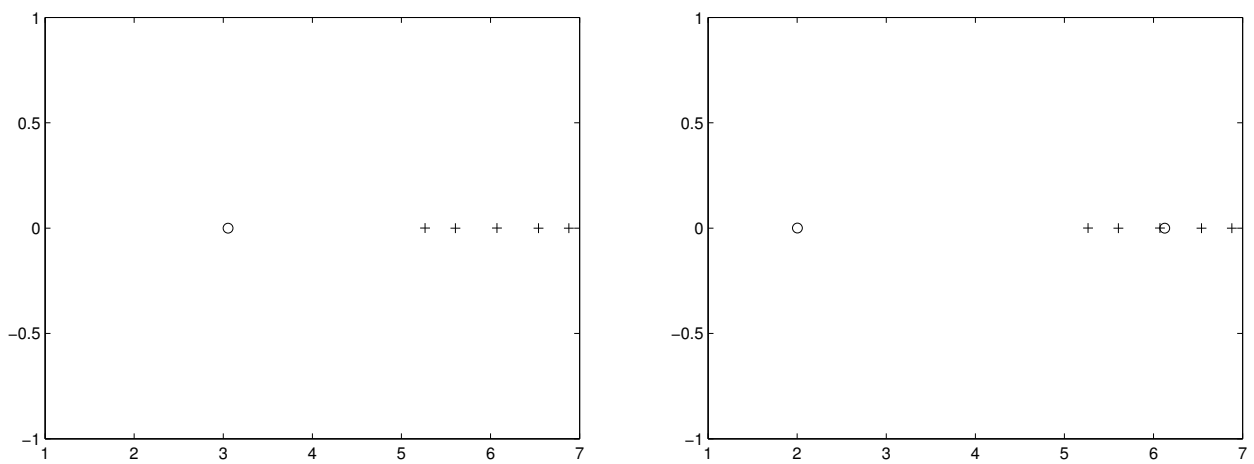

FIG. 5.5. Spectrum (plusses) and ordinary Ritz values (circles) in iterations one (left) and two (right) with GMRES applied to $H$ and $e_{1}$.
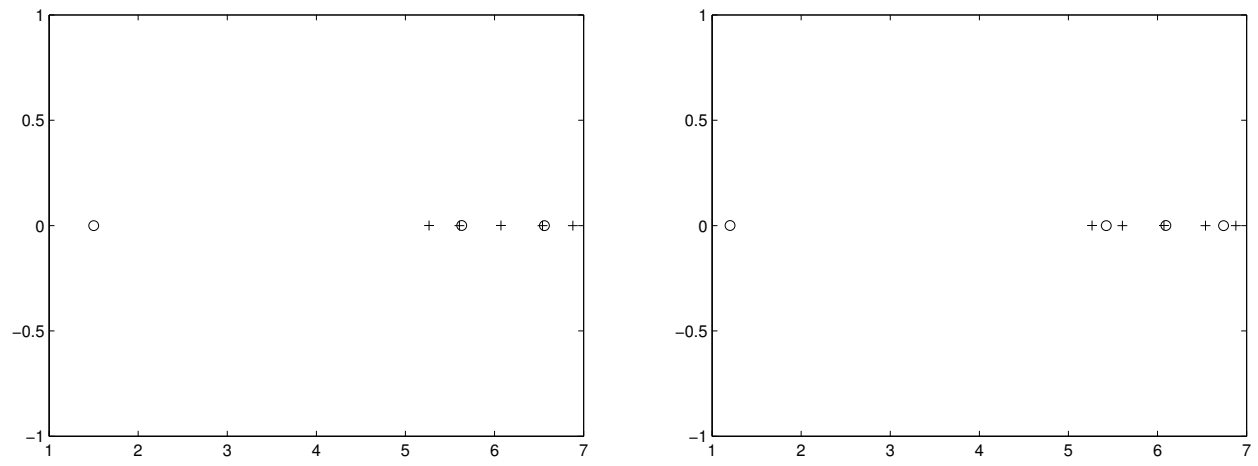

FIG. 5.6. Spectrum (plusses) and ordinary Ritz values (circles) in iterations three (left) and four (right) with GMRES applied to $H$ and $e_{1}$.
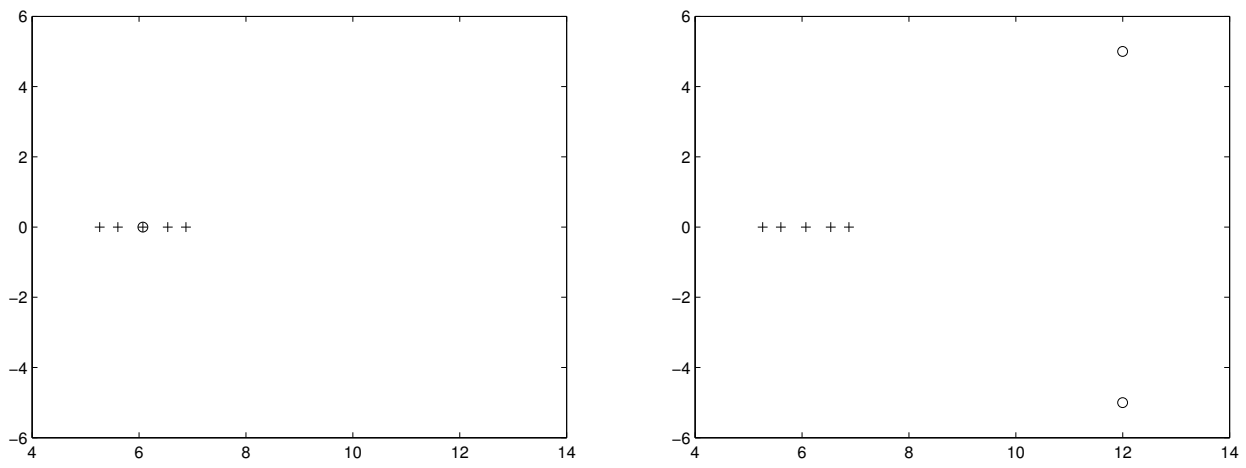

FIG. 5.7. Spectrum (plusses) and harmonic Ritz values (circles) in iterations one (left) and two (right) with GMRES applied to $\hat{H}$ and $e_{1}$. 

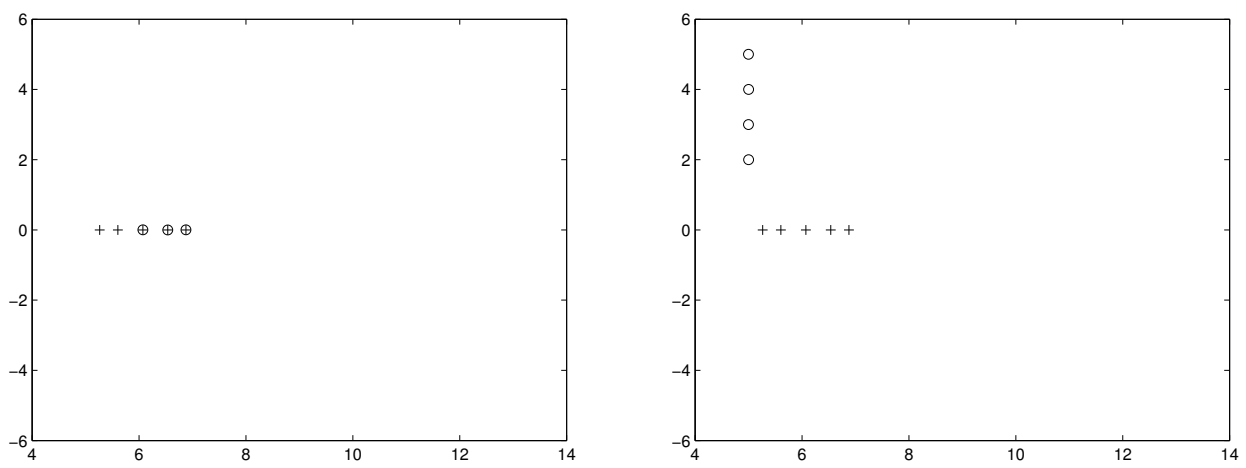

FIG. 5.8. Spectrum (plusses) and harmonic Ritz values (circles) in iterations three (left) and four (right) with GMRES applied to $\hat{H}$ and $e_{1}$.
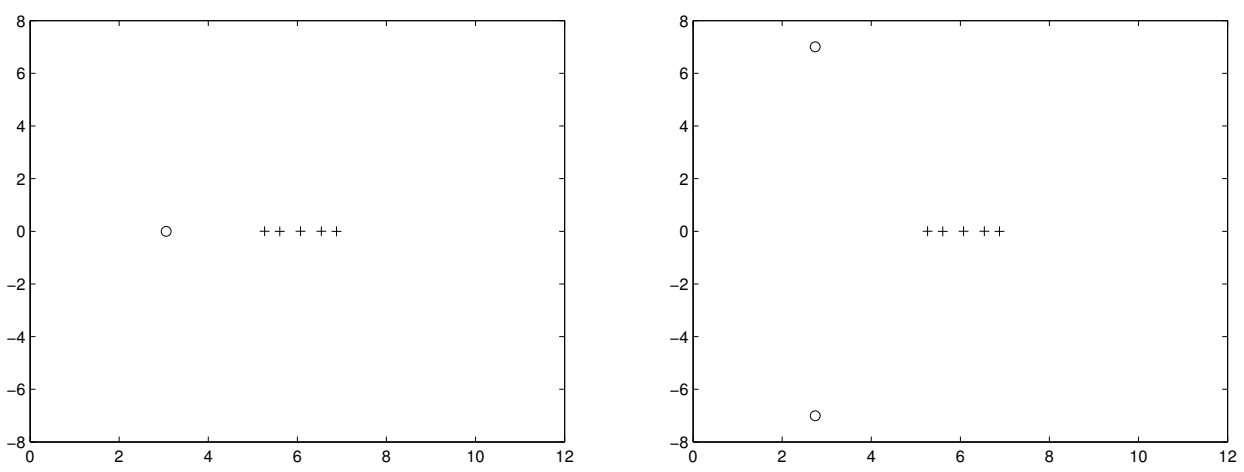

FIG. 5.9. Spectrum (plusses) and ordinary Ritz values (circles) in iterations one (left) and two (right) with GMRES applied to $\hat{H}$ and $e_{1}$.

The upper Hessenberg matrix $\hat{H}$ generating with $e_{1}$ such harmonic Ritz values and the residual norms (5.1) is

$$
\hat{H}=\left[\begin{array}{ccccc}
3.0541 & -16.1873 & -10.0627 & -35.309-45.281 i & -88.268+97.75 i \\
3.0359 & 2.4280 & 1.5833 & -4.692-12.494 i & -34.712+15.306 i \\
0 & 18.5186 & 12.7967 & 38.158+40.78 i & 94.067-99.876 i \\
0 & 0 & 1.2108 & 4.737+11.131 i & 15.109-4.888 i \\
0 & 0 & 0 & 4.162 & 7.345-11.131 i
\end{array}\right] \text {, }
$$

with $\left\|W^{*} W-I_{5}\right\| \approx 3,999$, where we rounded to three decimal places. The ordinary Ritz values of this matrix $\hat{H}$, behaving somewhat counter-intuitively as well, are displayed in Figures 5.9 and 5.10.

Finally, Theorem 4.1 also shows how to generate the same non-converging harmonic Ritz values from the original convection-diffusion problem, see Figures 5.3 and 5.4, but at the same time, force faster, linear convergence of GMRES residual norms, e.g.,

$$
\left\|r_{0}\right\|=1, \quad\left\|r_{1}\right\|=0.1, \quad\left\|r_{2}\right\|=0.01, \quad\left\|r_{3}\right\|=0.001, \quad\left\|r_{4}\right\|=0.0001 .
$$



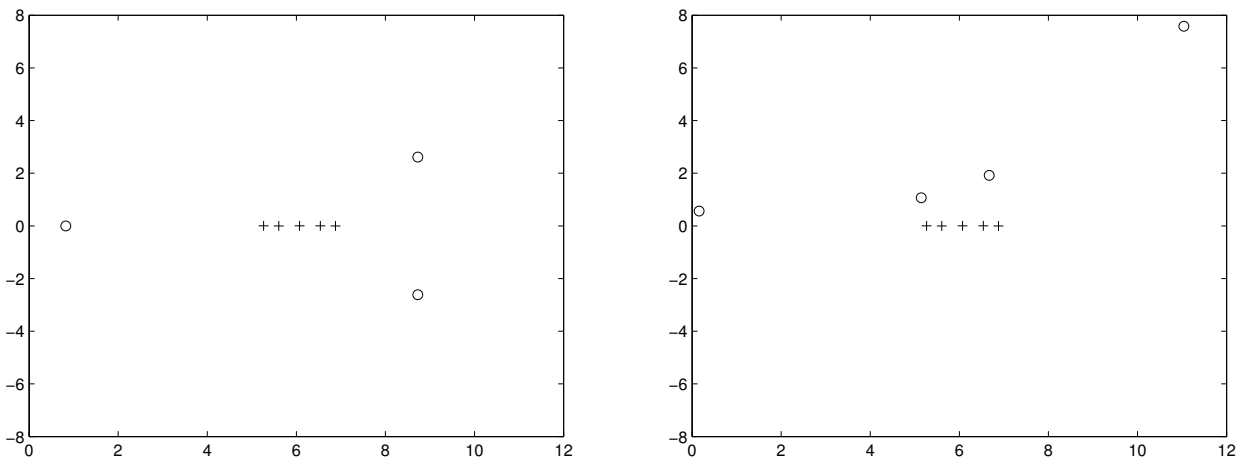

FIG. 5.10. Spectrum (plusses) and ordinary Ritz values (circles) in iterations three (left) and four (right) with GMRES applied to $\hat{H}$ and $e_{1}$.
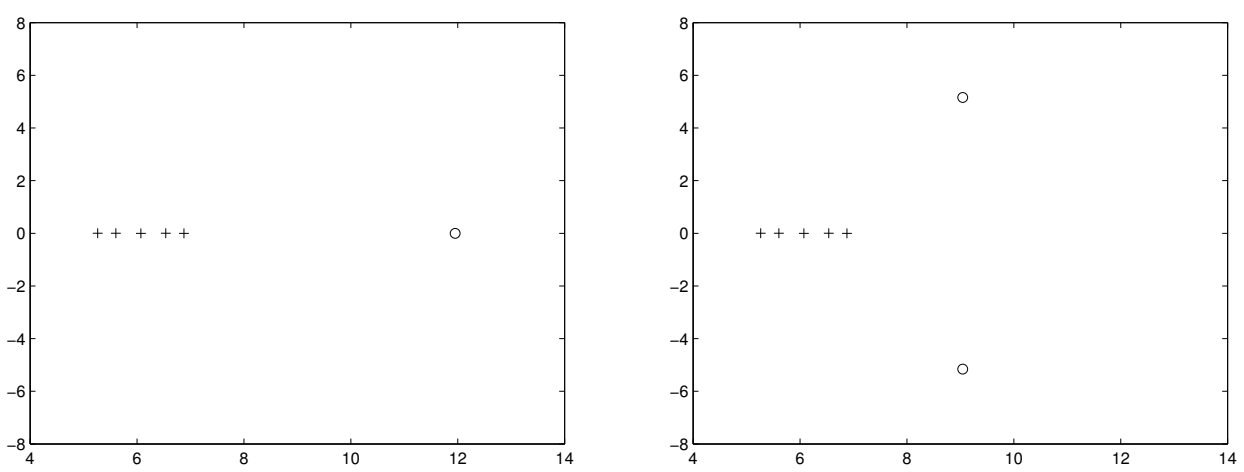

FIG. 5.11. Spectrum (plusses) and ordinary Ritz values (circles) in iterations one (left) and two (right) with GMRES applied to $\tilde{H}$ and $e_{1}$.

The upper Hessenberg system matrix $\tilde{H}$ for the corresponding constructed linear system is

$$
\tilde{H}=\left[\begin{array}{ccccc}
11.951 & -29.196 & 196.433 & -1474.331 & 11797.602 \\
1.201 & 6.138 & 0.005 & 1.747 & -13.995 \\
0 & 0.907 & 6.082 & 0.268 & -0.049 \\
0 & 0 & 0.806 & 6.077 & 0.884 \\
0 & 0 & 0 & 0.755 & 0.111
\end{array}\right]
$$

with $\left\|W^{*} W-I_{5}\right\| \approx 2.252$, where we again rounded to three decimal places. Even if this last matrix has some larger entries in its first row, its departure from normality as measured by $\left\|W^{*} W-I_{5}\right\|$ does not seem to deteriorate. The ordinary Ritz values of the matrix $\tilde{H}$ this time behave similarly to the harmonic Ritz values and are displayed in Figures 5.11 and 5.12.

6. Conclusion. In this paper we have shown that one can construct right-hand sides and matrices with a prescribed spectrum such that GMRES residual norms and harmonic Ritz values are also prescribed. This can be done with prescribed infinite harmonic Ritz values 


\section{ETNA}

Kent State University and

Johann Radon Institute (RICAM)
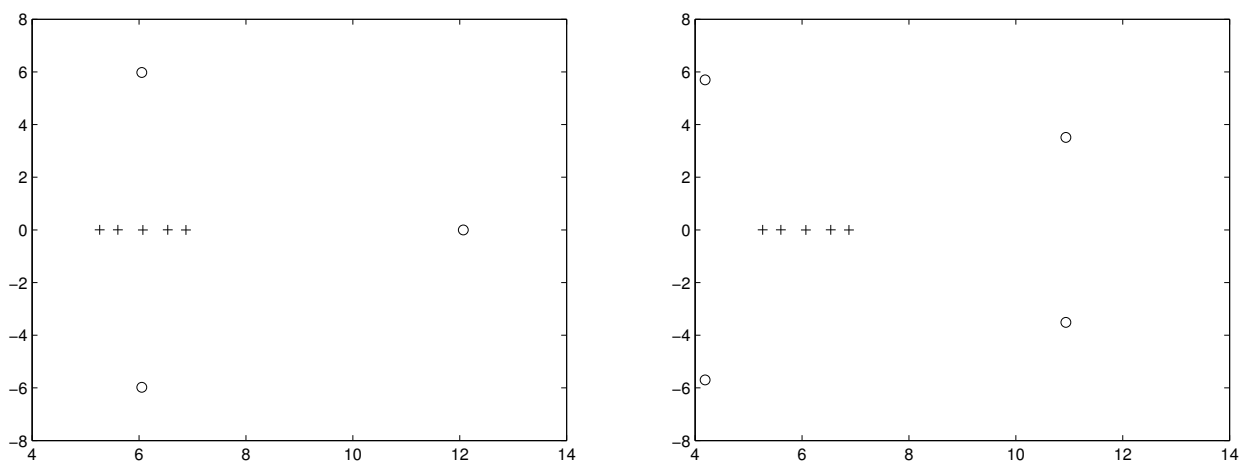

FIG. 5.12. Spectrum (plusses) and ordinary Ritz values (circles) in iterations three (left) and four (right) with GMRES applied to $\tilde{H}$ and $e_{1}$.

corresponding to prescribed stagnation iterations for GMRES. Hence, there need be no relation whatsoever between GMRES convergence and harmonic Ritz values.

The results described above raise some questions about the behavior of restarting methods for GMRES using deflation. There are many practical examples where using the harmonic Ritz vectors improves the convergence of restarted GMRES; see [34]. It will be interesting to find theoretical reasons for the fact that these deflation methods work in many cases despite of our results showing that there need be no relationship between GMRES convergence and harmonic Ritz values.

Software. At http://www.cs.cas.cz/duintjertebbens/duintjertebbens_soft.html the reader can find MATLAB functions to create matrices and initial vectors with the parametrization in this paper.

Acknowledgements. The research of Kui Du was supported by the National Natural Science Foundation of China (11771364 and 91430213), the Doctoral Fund of Ministry of Education of China (20120121120020), and the Fundamental Research Funds for the Central Universities (20720160002). The work of Jurjen Duintjer Tebbens was supported by the institutional support RVO:67985807 and by the grant GA13-06684S of the Grant Agency of the Czech Republic.

\section{REFERENCES}

[1] M. ARioli, V. PTÁk, AND Z. STRAKoš, Krylov sequences of maximal length and convergence of GMRES, BIT, 38 (1998), pp. 636-643.

[2] W. E. ARNOLDI, The principle of minimized iteration in the solution of the matrix eigenvalue problem, Quart. Appl. Math., 9 (1951), pp. 17-29.

[3] J. Baglama, D. Calvetti, G. H. Golub, and L. Reichel, Adaptively preconditioned GMRES algorithms, SIAM J. Sci. Comput., 20 (1998), pp. 243-269.

[4] P. N. BRown, A theoretical comparison of the Arnoldi and GMRES algorithms, SIAM J. Sci. Statist. Comput., 12 (1991), pp. 58-78.

[5] Z.-H. CAO, A note on the convergence behavior of GMRES, Appl. Numer. Math., 25 (1997), pp. 13-20.

[6] B. CARPENTIERI, I. S. DUFF, AND L. GiRAUd, A class of spectral two-level preconditioners, SIAM J. Sci. Comput., 25 (2003), pp. 749-765.

[7] B. CARPEntieri, L. GIRAUD, AND S. GRATTON, Additive and multiplicative two-level spectral preconditioning for general linear systems, SIAM J. Sci. Comput., 29 (2007), pp. 1593-1612.

[8] A. CHAPMAN AND Y. SAAD, Deflated and augmented Krylov subspace techniques, Numer. Linear Algebra Appl., 4 (1997), pp. 43-66. 
[9] J. Duintjer Tebbens and G. Meurant, Any Ritz value behavior is possible for Arnoldi and for GMRES, SIAM J. Matrix Anal. Appl., 33 (2012), pp. 958-978.

[10] - Prescribing the behavior of early terminating GMRES and Arnoldi iterations, Numer. Algorithms, 65 (2014), pp. 69-90.

[11] - On the convergence of Q-OR and Q-MR Krylov methods for solving nonsymmetric linear systems, BIT, 56 (2016), pp. 77-97.

[12] M. Eiermann, Fields of values and iterative methods, Linear Algebra Appl., 180 (1993), pp. 167-197.

[13] M. EIERMANN AND O. G. ERNST, Geometric aspects of the theory of Krylov subspace methods, Acta Numer., 10 (2001), pp. 251-312.

[14] M. EIERMANN, O. G. ERNST, AND O. SChNEIDER, Analysis of acceleration strategies for restarted minimal residual methods, J. Comput. Appl. Math., 123 (2000), pp. 261-292.

[15] J. ERhel, K. Burrage, AND B. Pohl, Restarted GMRES preconditioned by deflation, J. Comput. Appl. Math., 69 (1996), pp. 303-318.

[16] R. W. FREUnd, Quasi-kernel polynomials and their use in non-Hermitian matrix iterations, J. Comput. Appl. Math., 43 (1992), pp. 135-158.

[17] L. GIRAUD, S. GRATTON, AND E. MARTIN, Incremental spectral preconditioners for sequences of linear systems, Appl. Numer. Math., 57 (2007), pp. 1164-1180.

[18] L. Giraud, S. GRATTON, X. PINEL, AND X. VASSEUR, Flexible GMRES with deflated restarting, SIAM J. Sci. Comput., 32 (2010), pp. 1858-1878.

[19] S. GoossEnS AND D. Roose, Ritz and harmonic Ritz values and the convergence of FOM and GMRES, Numer. Linear Algebra Appl., 6 (1999), pp. 281-293.

[20] A. GReEnbaum, Generalizations of the field of values useful in the study of polynomial functions of a matrix, Linear Algebra Appl., 347 (2002), pp. 233-249.

[21] A. GReEnbaum, V. PTÁK, AND Z. STRAKOš, Any nonincreasing convergence curve is possible for GMRES, SIAM J. Matrix Anal. Appl., 17 (1996), pp. 465-469.

[22] A. Greenbaum AND Z. StRakoš, Matrices that generate the same Krylov residual spaces, in Recent Advances in Iterative Methods, G. Golub, A. Greenbaum, and M. Luskin, eds., vol. 60 of IMA Vol. Math. Appl., Springer, New York, 1994, pp. 95-118.

[23] M. R. Hestenes AND E. STIEFEL, Methods of conjugate gradients for solving linear systems, J. Research Nat. Bur. Standards, 49 (1952), pp. 409-436 (1953).

[24] M. Huhtanen AND O. NevanlinNa, Minimal decompositions and iterative methods, Numer. Math., 86 (2000), pp. 257-281.

[25] S. A. KHARCHENKO AND A. Y. YEREMIN, Eigenvalue translation based preconditioners for the GMRES(k) method, Numer. Linear Algebra Appl., 2 (1995), pp. 51-77.

[26] A. B. J. KUIJLAARs, Convergence analysis of Krylov subspace iterations with methods from potential theory, SIAM Rev., 48 (2006), pp. 3-40.

[27] C. Le CALVEZ AND B. Molina, Implicitly restarted and deflated GMRES, Numer. Algorithms, 21 (1999), pp. 261-285.

[28] D. Loghin, D. Ruiz, AND A. Tounami, Adaptive preconditioners for nonlinear systems of equations, J. Comput. Appl. Math., 189 (2006), pp. 362-374.

[29] G. Meurant, GMRES and the Arioli, Pták, and Strakoš parametrization, BIT, 52 (2012), pp. 687-702.

[30] — The coefficients of the FOM and GMRES residual polynomials, SIAM J. Matrix Anal. Appl., 38 (2017), pp. 96-117.

[31] G. Meurant And J. DuintJer TebBens, The role eigenvalues play in forming GMRES residual norms with non-normal matrices, Numer. Algorithms, 68 (2015), pp. 143-165.

[32] R. B. Morgan, A restarted GMRES method augmented with eigenvectors, SIAM J. Matrix Anal. Appl., 16 (1995), pp. 1154-1171.

[33] - Implicitly restarted GMRES and Arnoldi methods for nonsymmetric systems of equations, SIAM J. Matrix Anal. Appl., 21 (2000), pp. 1112-1135.

[34] - GMRES with deflated restarting, SIAM J. Sci. Comput., 24 (2002), pp. 20-37.

[35] N. M. Nachtigal, S. C. Reddy, AND L. N. TREFethen, How fast are nonsymmetric matrix iterations?, SIAM J. Matrix Anal. Appl., 13 (1992), pp. 778-795.

[36] C. C. PAige, B. N. PARlett, AND H. A. VAN Der Vorst, Approximate solutions and eigenvalue bounds from Krylov subspaces, Numer. Linear Algebra Appl., 2 (1995), pp. 115-133.

[37] C. C. PAIGE AND M. A. SAUNDERS, LSQR: an algorithm for sparse linear equations and sparse least squares, ACM Trans. Math. Software, 8 (1982), pp. 43-71.

[38] M. L. Parks, E. De Sturler, G. Mackey, D. D. Johnson, And S. Maiti, Recycling Krylov subspaces for sequences of linear systems, SIAM J. Sci. Comput., 28 (2006), pp. 1651-1674.

[39] J. Pestana AND A. J. WATHEN, On the choice of preconditioner for minimum residual methods for nonHermitian matrices, J. Comput. Appl. Math., 249 (2013), pp. 57-68.

[40] Y. SAAD, The Lanczos biorthogonalization algorithm and other oblique projection methods for solving large unsymmetric systems, SIAM J. Numer. Anal., 19 (1982), pp. 485-506. 
[41] Y. SAAD AND M. H. SCHULTZ, GMRES: a generalized minimal residual algorithm for solving nonsymmetric linear systems, SIAM J. Sci. Statist. Comput., 7 (1986), pp. 856-869.

[42] M. STYnes, Steady-state convection-diffusion problems, Acta Numer., 14 (2005), pp. 445-508.

[43] J. M. TANG, S. P. MACLACHLAN, R. NABBEN, AND C. VUIK, A comparison of two-level preconditioners based on multigrid and deflation, SIAM J. Matrix Anal. Appl., 31 (2009/10), pp. 1715-1739.

[44] J. M. TANG, R. NABben, C. Vuik, AND Y. A. ERlangGa, Comparison of two-level preconditioners derived from deflation, domain decomposition and multigrid methods, J. Sci. Comput., 39 (2009), pp. 340-370.

[45] L. N. TRefethen AND D. BAU, III, Numerical Linear Algebra, SIAM, Philadelphia,1997.

[46] L. N. Trefethen And M. Embree, Spectra and Pseudospectra, Princeton University Press, Princeton, 2005.

[47] A. VAN DER SLUIS AND H. A. VAN DER VORST, The rate of convergence of conjugate gradients, Numer. Math., 48 (1986), pp. 543-560.

[48] H. A. VAN DER VORST AND C. VUIK, The superlinear convergence behaviour of GMRES, J. Comput. Appl. Math., 48 (1993), pp. 327-341. 